\title{
Dynamic light scattering distributions by any means
}

\author{
Natalia Farkas 1 - John A. Kramar (D)
}

Received: 20 February 2021 / Accepted: 22 April 2021 / Published online: 21 May 2021

(C) The Author(s) 2021

\begin{abstract}
Dynamic light scattering (DLS) is an essential technique for nanoparticle size analysis and has been employed extensively for decades, but despite its long history and popularity, the choice of weighting and mean of the size distribution often appears to be picked ad hoc to bring the results into agreement with other methods and expectations by any means necessary. Here, we critically discuss the application of DLS for nanoparticle characterization and provide much-needed clarification for ambiguities in the mean-value practice of commercial DLS software and documentary standards. We address the misleading way DLS size distributions are often presented, that is, as a logarithmically scaled histogram of measured relative quantities. Central values obtained incautiously from this representation often lead to significant interpretation errors. Through the measurement of monomodal nanoparticle samples having an extensive range of sizes (5 to $250 \mathrm{~nm}$ ) and polydispersity, we similarly demonstrate that the default outputs of a frequently used DLS inversion method are ill chosen, as they are regularizer-dependent and significantly deviate from the cumulant $z$-average size. The resulting discrepancies are typically larger than $15 \%$ depending on the polydispersity index of the samples. We explicitly
\end{abstract}

N. Farkas

Theiss Research, 7411 Eads Ave, La Jolla, CA 92037, USA

N. Farkas $(\bowtie) \cdot$ J. A. Kramar

National Institute of Standards and Technology, Gaithersburg, MD 20899, USA

e-mail: natalia.farkas@nist.gov identify and validate the harmonic mean as the central value of the intensity-weighted DLS size distribution that expresses the inversion results consistently with the cumulant results. We also investigate the extent to which the DLS polydispersity descriptors are representative of the distributional quality and find them to be unreliable and misleading, both for monodisperse reference materials and broad-distribution biomedical nanoparticles. These results overall are intended to bring essential improvements and to stimulate reexamination of the metrological capabilities and role of DLS in nanoparticle characterization.

Keywords Dynamic light scattering · Harmonic mean NNLS inversion - Size distribution · Moment-ratio transformation $\cdot$ Polydispersity index $\cdot$ Nanoparticles

\section{Introduction}

The significance of nanoparticles is apparent and indisputable in a broad range of scientific and technical fields. They substantially enhance the performance of many industrial and research-oriented products. Successful realization of such products is critically dependent on reliable dimensional measurements to correlate nanometer-scale physical-chemical properties with their intended functionality. Dynamic light scattering (DLS) is the method of choice in most cases. In recent years, for instance, more than $50 \%$ of drug products containing nanomaterials submitted to the Center for Drug Evaluation and Research within the US Food and Drug 
Administration relied on DLS for size characterization (D'Mello et al. 2017). Despite its overwhelming dominance in nanoparticle characterization, consistency and reproducibility of the DLS measurements remain a significant challenge, mainly because of questionable data analysis and interpretation.

In current practice, the choice of weighting and means of the size distribution by DLS is often unjustified or appears to be picked ad hoc (Bazylińska 2017; Myerson et al. 2018; Takechi-Haraya et al. 2018; Chen et al. 2017; DeLoid et al. 2017). Ambiguities in mean-value terminology are also prevalent in commercial DLS software and documentary standards (Zetasizer nano user manual 2013; ASTM E2490-09:2015; ISO 22412:2017). A common dilemma is that the DLS software typically offers several types of central values based on intensity, volume, or number weighting as the output of the measurement (Bhattacharjee 2016). Unfortunately, however, instrument manufacturers (Nobbmann and Morfesis 2009), best practice guides (Nicolas et al. 2018), and documentary standards (ASTM E2490-09:2015; ISO 22412:2017) frequently offer vague, inadequate, or even incorrect guidance. For instance, the definition of the DLS size in the current ISO 22412 (2017) as the "central value of the underlying particle size distribution" is ambiguous, i.e., whether it refers to the mean, median, mode, or other central tendencies. Thus, the nano-bio community is left with insufficient guidelines which lead to serious data analysis and interpretation errors with implications beyond just irreproducibility, especially when the key conclusions of a study are critically dependent on such choices. Available review articles merely identify some of the deficiencies of DLS characterization and only briefly mention the risk of conversion error between weighted means (Bhattacharjee 2016). In fact, they are frequently cited as an explanation for disagreement with other methods or with expectations with no solution offered, even in cases where the inconsistent DLS results are simply due to questionable data analysis or the lack of metrological rigor.

Considering the topical urgency generated by the aforesaid state of DLS characterization practice for nano-bio materials in particular, we herein present a critical discussion of the data analysis and interpretation challenges affecting DLS dimensional metrology. We provide a complete and systematic articulation of how these mathematical and statistical measurement issues could lead to significant and often unrecognized errors. Sample and sample preparation related measurement limitations, such as multiple-scattering effects, particle- particle interactions, heterogeneity in particle size, shape, and composition, are adequately described in the literature (ISO 22412:2017; Bhattacharjee 2016) and will not be considered here. First, we address the misleading way DLS size distributions are often presented, i.e., as a histogram of measured relative quantities based on a logarithmic scale. Obtaining central values incautiously from such graphical representation leads to significant interpretation errors. Based on extensive measurements, we also demonstrate that the default output of the non-negatively constrained leastsquares (NNLS) inversion method given by common DLS analysis software is ill chosen, since it is regularizer-dependent and significantly deviates from the cumulant $z$-average size. The resulting errors are typically larger than $15 \%$ depending on the polydispersity index (PDI) of the samples. We explicitly identify and validate the harmonic mean as the physicalmeasurement-justified central value of the intensityweighted DLS size distribution. Quantitative agreement between the cumulant and inversion results originating from the same DLS data is demonstrated. Importantly, we investigate the extent to which the DLS polydispersity descriptors are representative of the distributional quality and find them to be completely unreliable and misleading, both for reference materials and biomedical nanoparticles. In summary, the results critically advocate for essential improvements in existing DLS data interpretation practices that behoove strong consideration for adoption by the nano-bio community, regulatory agencies, and instrument manufacturers.

\section{Results and discussion}

\section{The DLS measurement process}

A DLS measurement samples laser light scattered from the simultaneous Brownian motion of a large ensemble of particles in solution. The fundamental quantity measured is the time autocorrelation function (ACF) of the scattered light intensity. Decay rates for the ensemble of particles are embedded within the ACF and are related to an apparent mean diffusion constant, which is then converted to particle diameter through the StokesEinstein equation. Since the diameter is derived from scattered intensity and is inversely proportional to the decay rate, the DLS-measured average hydrodynamic size is naturally presented as an intensity-weighted 
harmonic mean. There are two approaches commonly used to analyze the ACF: cumulant analysis and direct inversion-typically an NNLS inversion method (Zetasizer nano user manual 2013). In the cumulant method, the intensity-weighted, harmonic-averaged diameter is referred to as the $z$-average and is calculated from the first cumulant of the ACF. A distributional width parameter, the PDI, is derived from a ratio of the first and second cumulants. The NNLS approach poses a set of particle radii and the corresponding set of decay rates and performs a least-squares fitting to obtain the weightings for a logarithmically scaled histogram of particle sizes. The theory and practice of the cumulant method are generally well documented and provide deterministic and reliable size estimates for particle samples with a coefficient of variation (CV) less than $3 \%$ in an appropriate solution environment in terms of dilution and salt concentration. Inversion methods, on the other hand, are mathematically ill posed and highly dependent on the inversion parameters.

\section{Parametrization and graphical representation of DLS} data

To understand the analysis of DLS data and avoid confusion over "which mean do you mean?", we must first address the easily misunderstood way NNLS size distributions are characteristically presented, that is, as logarithmically scaled relative quantities displayed as a histogram. Simply changing the logarithmic scale to linear is incorrect, as is obtaining central values directly from such graphical representations (Hess 2004). These practices are, however, frequent in current DLS studies (DeLoid et al. 2017; Yeap et al. 2018; Maguire et al. 2018) and often lead to questionable study results. To prevent interpretation errors, the logarithmically spaced discrete data - the typical output of NNLS softwareneeds to be transformed to a linearly scaled density distribution as described in ISO 9276-1 (1998).We show an example of a correct transformation for the output from a NNLS inversion method analysis in Fig. 1a. Here, we utilize as a mathematical convenience the model assumption of a lognormal distribution, in terms of a scale-parameter $(\exp (\mu))$ and a shape-parameter $(\sigma)$ related to the distribution width; however, the described general trends in mean transformations apply to all monomodal distributions. The lognormal is appropriate here since both the NNLS-output distributions and typical actual particle size distributions are in general (Kiss et al. 1999) well-approximated by such a form. Fig. 1a demonstrates that reading off the mode from the logarithmically scaled histogram of the measured relative quantities leads to an overestimation of the actual value by a factor of $\exp \left(\sigma^{2}\right)$, which specifically translates to a $10 \%$ error in the case of this particular typical sample of 100-nm mean-diameter gold nanoparticles.

It is generally accepted that the NNLS distributions are broad due to the data weighting and smoothing necessary for dealing with the inherent noise in the ACF during the inversion process (Xu et al. 2018), but the fact that the broadness of the distribution is regularizer-dependent is largely ignored. With simple modification of the regularizer value (or other inversion parameters, such as the size range and the number of bins), very different size distributions may be produced from the same ACF data. This is demonstrated for the 100-nm gold nanoparticle sample in Fig. 1b where a single set of ACF data is analyzed using a range of parameters in the inversion algorithm. With prior knowledge, it is possible to achieve good agreement between the NNLS distribution and atomic force microscope (AFM) size distribution (transformed to intensityweighted for comparison). However, without prior knowledge, it is impossible to confidently obtain the correct width of the distribution from DLS measurements (i.e., the shape-parameter in the case of a lognormal distribution). If left uncorrected, the impact of an incorrect-almost always too large-shape-parameter is amplified dramatically during conversions between weighted means (ISO 9276-2:2014), which has important consequences for data interpretation.

Width-error and its consequence on transformation

We show in Fig. 2 that the typical positive error in the shape-parameter, which we refer to as width-error, will almost always lead to significant underestimation when transforming from the intensity-weighted to the volumeand number-weighted central values of the distribution. The calculated central values of the transformed NNLS number-weighted distribution wildly undershoot the corresponding size estimates obtained by microscopy even for certified reference particles with narrow distributions. For example, this discrepancy has been reported in a recent study involving unilamellar liposomes (Barberio et al. 2020). In this case, the number-weighted arithmetic mean is shown to underestimate the corresponding $z$-average size of the liposomes by as much as $35 \%$. Using the 


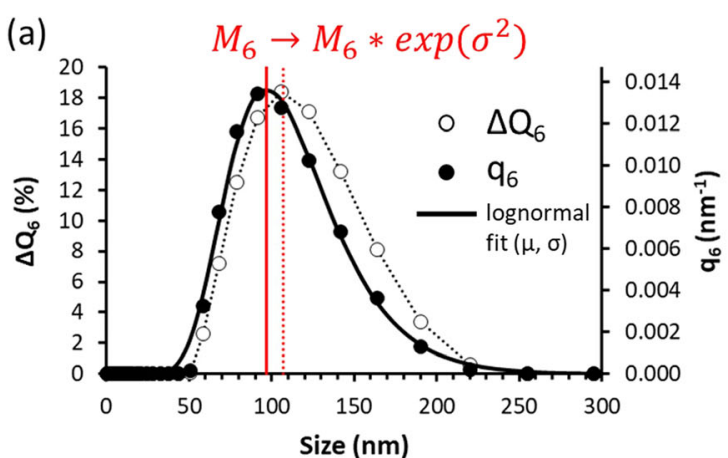

Fig. 1 a Graphical representation of the intensity-weighted size data obtained for 100-nm gold nanoparticles by the NNLS inversion method. Histograms are of the measured relative quantities $\left(\Delta \mathrm{Q}_{6}\right)$ (open circles) and the corresponding density distribution $\left(\mathrm{q}_{6}\right)$ (solid circles) calculated by dividing the relative quantities by the corresponding logarithmically scaled size intervals. The solid line is a lognormal fit, in terms of a scale-parameter $(\exp (\mu))$ and a shape-parameter $(\sigma)$, to the density distribution. The dotted line is only to guide the eye between the discrete NNLS data points. Note that the most frequently occurring size class in the logarithmically scaled histogram of the relative quantities differs from the actual mode $(\mathrm{M})$ of the size distribution by a factor of $\exp \left(\sigma^{2}\right)$. b Comparison of intensity-weighted density distributions obtained for 100-nm gold nanoparticles by AFM (dotted line) and DLS (solid

DLS-measured diameter as an input, the study proposes to estimate the number of lipid and cytokine molecules per liposome. However, given the findings in Fig. 2, the choice of the number-weighted average size in this particular work would introduce a bias in the calculated results. The latest revisions of ASTM (ASTM E3247-20:2020) and ISO (ISO 22412:2017) standards specifically deprecate the use of number distributions, especially for methods involving smoothing in the process of extracting the intensity-weighted size distribution. Although acknowledged, the warning is often ignored by the measurement community (Coleman et al. 2011; Di Francesco and Borchard 2018; Liu et al. 2019; Ulasov et al. 2011; Chen et al. 2019). One of the arguments is that the effect of the shape-parameter on the transformation from intensity to volume or number is minimal for particles with narrow size distributions (ASTM E2490-09:2015). This is certainly the case when the shape-parameter reflects the actual narrow distribution as it does for the microscopy data, but definitely not for the vast majority of DLS-derived estimates where substantial width-error is unequivocally present when using default parameters. For instance, the difference between number- and intensity-weighted arithmetic means calculated from the size distribution of NIST Standard Reference Material ${ }^{\circledR}$ SRM 1963 (National Institute of Standards and Technology 2003) we obtain

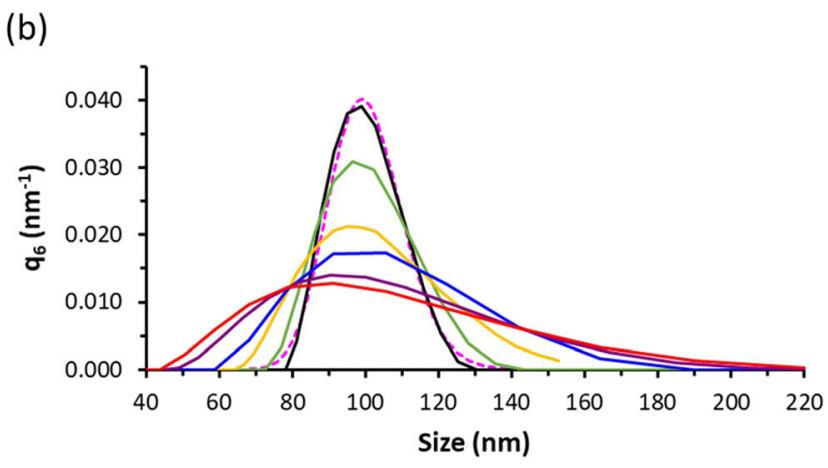

lines). The AFM distribution was transformed from number to intensity-weighted and fitted with a lognormal function. All of the plotted DLS distributions (solid lines in different colors) are generated from the same ACF measurement data with only a readjustment of the parameters of the NNLS inversion algorithm. The red line (flattest curve) represents the density distribution obtained with the default "general purpose" algorithm from a commonly used instrument $(0.4 \mathrm{~nm}$ to $10 \mu \mathrm{m}$ size range, 70 size bins, 0.01 regularizer value). Good agreement is achievable between the NNLS and AFM size distributions, but only by the contortions of limiting the NNLS-inversion parameters to a size range of 10 to $500 \mathrm{~nm}$ and using 100 size bins and a regularizer value of 0.001 (black line)

by microscopy is only $0.2 \%$, while it is $25 \%$ when calculated from the NNLS-derived distribution using a typical default "general purpose" algorithm (Fig. 2). The larger the width-error, the broader the size range that is obtainable by choosing different weightings, even for ideal standard particles. Ad hoc or unjustified selection of these size values to align with other methods is seemingly a common practice especially in the case of industrial and pharmaceutical materials (Bazylińska 2017; Myerson et al. 2018; Takechi-Haraya et al. 2018; Chen et al. 2017; DeLoid et al. 2017).

Historically, the effect of the width-error was first encountered in the work of Thomas over 30 years ago (Thomas 1987), before the widespread use of inversion methods. Thomas proposed to calculate the numberweighted arithmetic mean from the direct output of the cumulant analysis (i.e., the intensity-weighted $z$-average and PDI) with the key assumption that the relative variance of the distribution is equal to the cumulant PDI. The calculations largely underestimated the experimental mean size obtained by microscopy. Thomas speculated that the PDI might overestimate the relative variance of the distribution due to noise in the ACF, which led him to propose an empirical reduction of the PDI. A decade later, Hanus and Ploehn (1999) reinvestigated Thomas' approach with expanded data 


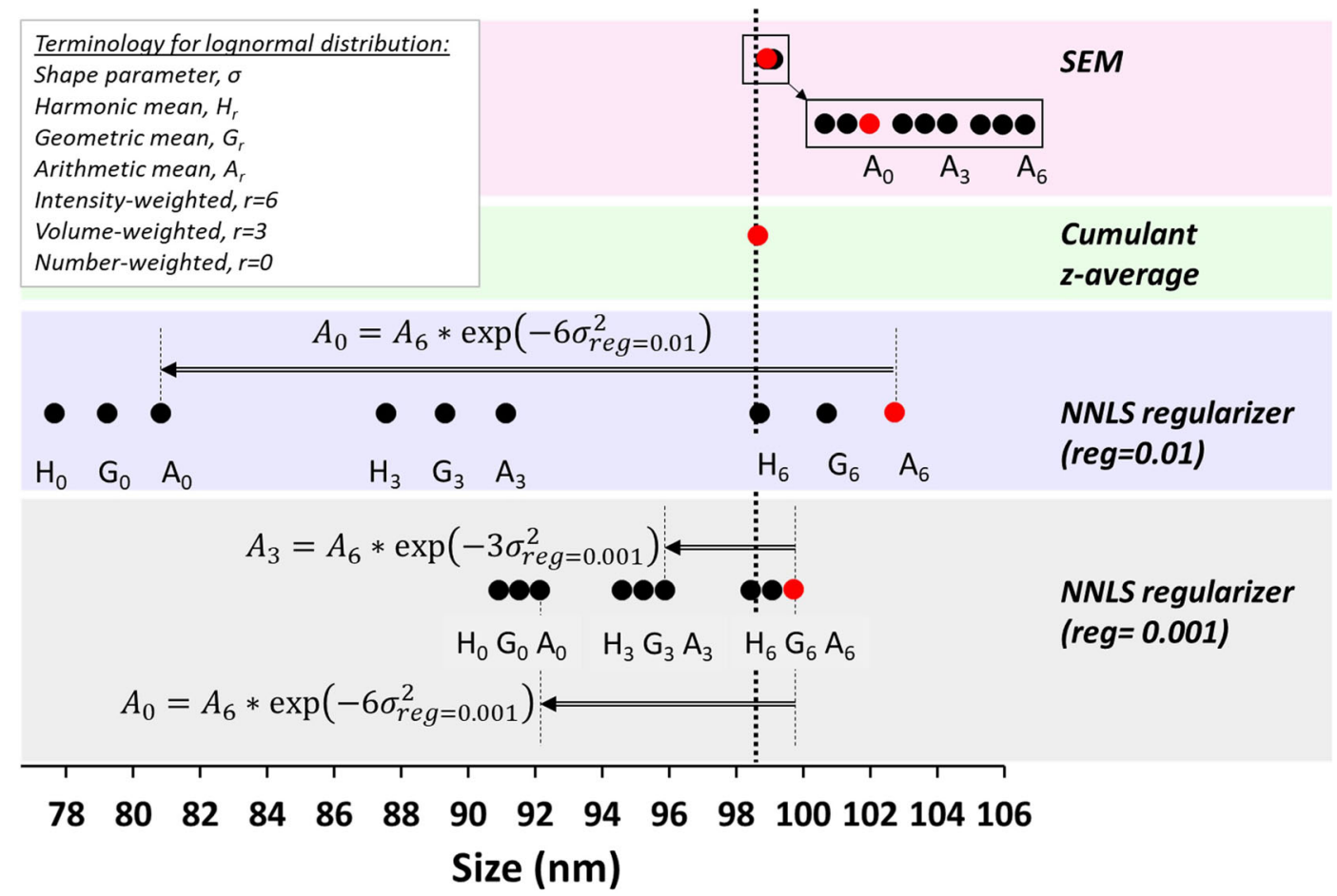

Fig. 2 Relationship between the differently weighted Pythagorean means calculated from the measured number-weighted SEM and intensity-weighted NNLS distributions. Red and black points indicate instrument output and calculated data, respectively. (In this case, the default DLS instrument output is the arithmetic mean.) This example is for SRM 1963 100-nm polystyrene nanoparticles. The density distributions are fitted by lognormal functions with shape-parameter, $\sigma$, which equals $0.017,0.2$, and 0.115 for the SEM, NNLS (reg $=0.01$ ), and NNLS (reg $=0.001$ )

sets and additional analytical effort, in essence reaching the same conclusion that a reduction of the PDI was necessary to properly fit their data. Unfortunately, amount of reduction in the PDI needed is not consistent between different cases. We note that the inconsistency between DLS-measured polydispersity and the relative variance of the actual distribution - which prompted the width-error correction in these historic DLS studiespersists in present-day implementations of the inversion method through the indeterminacy of the regularizer value and other inversion parameters.

Comparison of the outputs of the cumulant and NNLS inversion methods

While the width-error causes inaccuracies in transforming between weightings of the distributions, it also causes errors when comparing the different Pythagorean means. Our measurement and analysis of SRM 1963 with a CV distributions, respectively. The moment-ratio transformations between means possess an underlying scale-factor of $\exp \left(0.5 \sigma^{2}\right)$ from $\mathrm{H}$ to $\mathrm{G}$ and $\mathrm{G}$ to $\mathrm{A}$; transformations between weightings possess a further scale factor of $\exp \left(3 \sigma^{2}\right)$ from number to volume and volume to intensity. The larger the width-error, the more severe the underestimation of the volume- and numberweighted mean values from the default DLS-output NNLS-derived size distribution. The dotted line indicates values that are in agreement between methods

of $2 \%$ (Fig. 2) reveal that while the arithmetic mean of the NNLS-derived intensity-weighted size distribution depends on the regularizer and significantly deviates from the cumulant $z$-average and scanning electron microscopy (SEM) results, the harmonic mean in contrast does coincide irrespective of the regularizer value used. This is not unexpected since based on the underlying 1/D dependence of the Stokes-Einstein equation used for determining size from the $\mathrm{ACF}$, the mathematically natural output of the DLS method would be the harmonic mean of the intensity-weighted distribution. Unfortunately, one of the most popular software programs (Zetasizer nano user manual 2013) for the NNLS inversion method chooses as its default output the arithmetic mean. Since there is no requirement in international documentary standards for consistent representation of the cumulant and inversion methods, this unguided choice is consistent with standard practices, but not without negative implications for certain applications. A recently published work, for instance, 
proposes the combination of analytical ultracentrifugation and DLS to determine porosity of polyplex nanoparticles (Niebel et al. 2014). The DLS-measured size is one of the inputs meant to be an independent variable. The authors' choice for DLS size is the arithmetic mean of the intensity-weighted NNLS distribution seemingly unaware that it overestimates the $z$-average by at least $20 \%$ depending on the PDI of their samples. Such discrepancy may introduce significant unrecognized error/ uncertainty into their porosity calculation. Another work proposes the combination of DLS and TEM to determine the shell volume of nanoparticles as the difference between the hydrodynamic volume and the core volume (Chen et al. 2017). Here as well, the intensity-weighted arithmetic mean was unjustifiably used, in this case for the hydrodynamic diameter.

In our view, the effect of the inevitable width-error in DLS behooves the selection of the harmonic mean of the intensity-weighted size distribution for obtaining size values that are metrologically comparable between methods. To demonstrate that this is routinely possible, we measure a variety of monomodal nanoparticles with an extensive range of size and polydispersity including some of the most significant reference material typesi.e., polystyrene, silica, and gold - as well as functional polyplex nanoparticles (Farkas et al. 2019). The results of the cumulant and NNLS inversion methods for the 44 samples in total are summarized in Fig. 3. We find that the arithmetic mean of the NNLS intensity-weighted size distribution overestimates the cumulant $z$-average

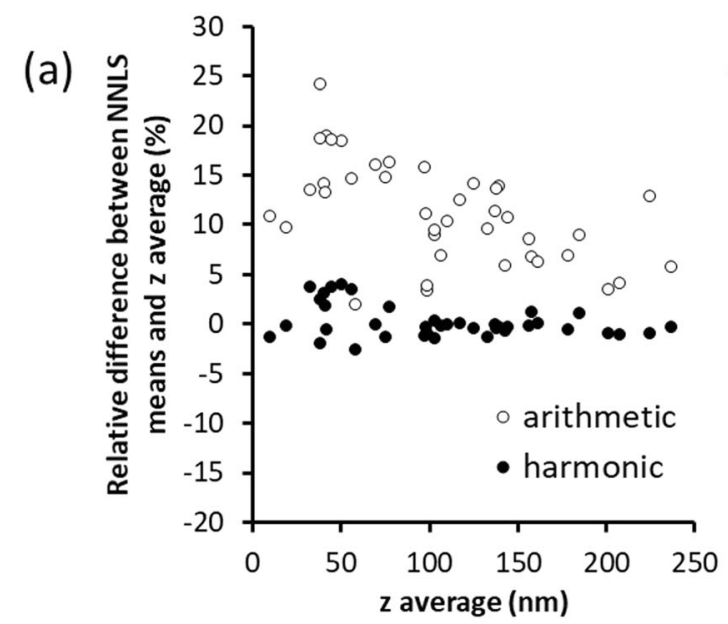

Fig. 3 a Relative differences between the intensity-weighted NNLS (reg $=0.01$ ) means (arithmetic or harmonic) and the cumulant $z$-average as a function of the $z$-average diameter for a large set of different nanoparticle types and sizes (polystyrene, in all cases, and the degree to which it does so is related to the PDI (Fig. 3b). The harmonic mean of the intensity-weighted size distribution, on the other hand, agrees with the $z$-average within $2 \%$ on average. This is significant because we can now state a definition of the DLS size that encompasses both the cumulant and distributional result in a consistent manner.

Polydispersity descriptors: are they good indicators of the broadness of the true distribution?

Having demonstrated the effect of the width-error on the mean value when transforming between weightings and Pythagorean means, our final goal in this work is to address the unjustified dependence on DLS to provide a serviceable indication of the distributional property of nanoparticle samples. In a recent study on quantifying the influence of nanoparticle polydispersity on cellular delivered dose (Johnston et al. 2018), for instance, polydispersity is defined as the shape-parameter of a lognormal function fitted to the intensity-weighted NNLS size distribution, which as we have shown is unreliable and always too broad. Such improper valuation of the polydispersity renders the quantification that is supposed to be the independent variable for the dose-delivery experiment questionable at best. Even recent editorials on best practices for nanoparticulate systems encourage the use of the intensity-weighted distribution "as it is considered always correct" (Nicolas et al. 2018). This is concerning because such a statement is misleading. As previously

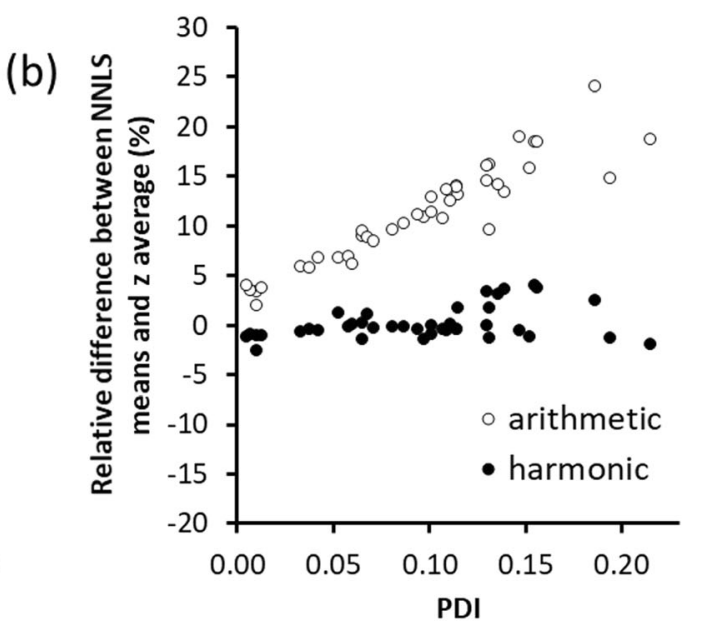

silica, gold, and polyplex nanoparticles in the 5 to $250 \mathrm{~nm}$ size range). b Same relative differences as a function of the PDI obtained from the cumulant method 
shown, the intensity-weighted distribution is indeed the correct one from which to calculate the harmonic mean, but in context, this statement appears to endorse reliance on the distribution itself, a practice all too often taken.

As we have shown above, the width of the NNLSdetermined intensity-weighted size distribution is neither quantitative nor qualitatively reliable, as it is regularizer-dependent. If alternatively using the cumulant analysis, the PDI is also known to significantly overestimate the relative variance of the actual size distribution (often by an order of magnitude) due to the inescapable need for ACF noise reduction by truncation (Koppel 1972), hence it is not a quantitative measure of polydispersity. But the PDI is at least deterministic, so does it provide at least a qualitative indication of the distributional property of nanoparticle samples? To investigate the question methodically, gold reference and functional polyplex nanoparticles in a broad range of size and polydispersity were measured by DLS and AFM. The ratio of the $z$-average and AFM size is correlated in Fig. 4a and b with the measures of polydispersity, i.e., the relative variance of the AFM number-weighted distribution (Q) and the PDI from the cumulant analysis. The deviation between DLS and AFM measurements would be expected to increase with increase of the actual polydispersity of the sample because the DLS-derived mean, originating from an intensity-weighted measurement which scales as the sixth power of the particle radius, is extremely sensitive to and skewed by the positive tail of the distribution. The gold nanoparticle suspensions are free from largesize outliers and aggregates and the nanoparticles exhibit a narrow size distribution with a relatively small Q that is roughly constant for the entire series.
Consequently, their AFM and DLS sizes agree on average. The polyplex nanoparticles in contrast have widely varying $\mathrm{Q}$, which is often the case for functional, multicomponent nanoparticles. We find that the increase in $\mathrm{Q}$ parallels the increase in the $z$-average/AFM size ratio, also as expected. In contrast, no discernable trend is observed when the same ratios are plotted as a function of PDI (Fig. 4b). Samples with low PDI may measure inconsistently by the two methods, while samples with high PDI may measure consistently. It becomes apparent in Fig. 4c that sensitivity of DLS to the tail of large particles significantly biases the size measurements by both the cumulant and NNLS inversion methods.

These observations present a major challenge for standardization in nanoparticle metrology. For example, the mean size of ERM-FD304, a certified colloidal silica nanoparticle suspension (Institute for Reference Materials and Measurements of the European Commission's Joint Research Centre 2012), can be measured consistently by DLS using both the cumulant and NNLS inversion methods when expressed as the intensityweighted harmonic mean, but the positive skewing in the size distribution renders its use very limited for traceability transfer to microscopy or to other individual sizing techniques. Furthermore, the findings in Fig. 4 demonstrate that PDI values are generally unreliable predictors of nanoparticle polydispersity. We see that both the mean and distributional DLS measurement results may be unknowably significantly biased, which would lead to ambiguous interpretation of structurefunction relationships of nanoparticle formulations or to inconclusive interlaboratory comparisons in terms of metrological compatibility. Cross-examination by a sizing method for individual particles is therefore a must

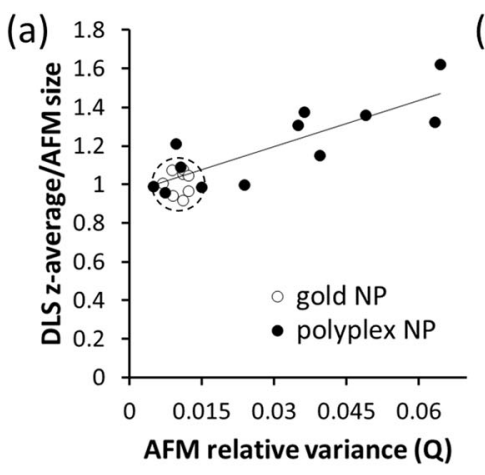

Fig. 4 a Correlation between the relative variance of the AFM number-weighted distribution (Q) and the ratio of DLS $z$-average and AFM sizes. $\mathbf{b}$ The ratio of DLS $z$-average and AFM sizes as a function of the PDI obtained from the cumulant method. c
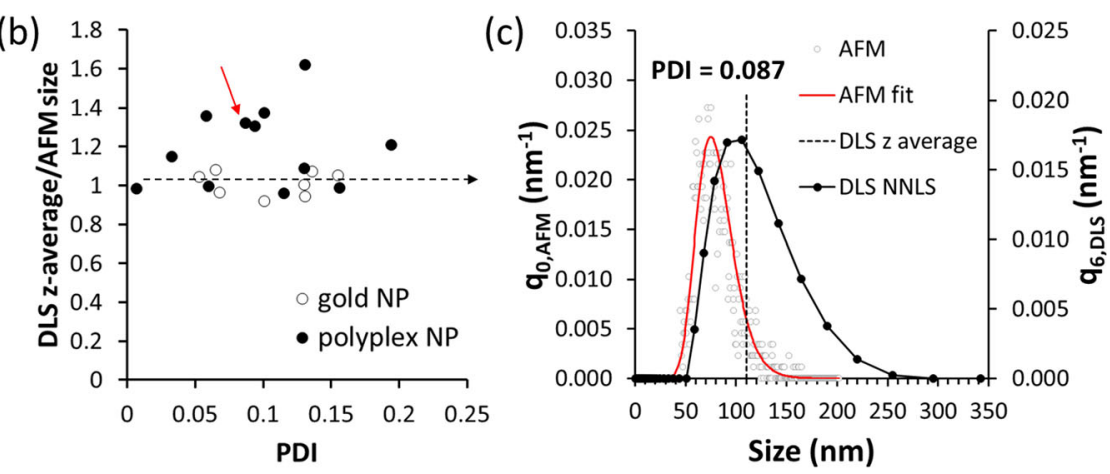

Distributional details of the AFM and DLS size measurements for the data point marked by the arrow in Fig. 4b. A PDI value of 0.1 or below is widely assumed to indicate a nearly monodisperse sample 
for credible reporting of nanoparticle size measurements (both mean size and distribution), and for reversing the alarming practice of the exclusive reliance on DLS-only in measurement-critical application fields.

\section{Conclusions}

In conclusion, we have investigated the nanoparticle size and size-distribution output results from DLS using both the cumulant and NNLS inversion data analysis methods and compared them with individual particle sizing techniques. The NNLS distributions are shown to be seriously influenced by excessive smoothing, which is unavoidable because of the mathematically ill-posed nature of the data inversion step. We resolve ambiguities associated with distribution functions and their Pythagorean means and demonstrate that the intensity-weighted harmonic mean is a simple and transparent way of reporting the output data that for monomodal samples generally renders a reliably consistent mean value. This definition addresses previous discrepancies between cumulant and NNLS inversion results for DLS and most significantly (from the perspective of measurement science) presents a single, consistent DLS-derived size, which can be arrived at using either analysis method. Polydispersity descriptors attainable from DLS measurements using either the inversion or cumulant methods were shown to be generally unreliable in terms of their serviceability in describing the distributional properties of both narrowdistribution standard particles and the more-typical broad-distribution biomedical nanoparticles. These results advocate for crucial refinements of existing DLS data analysis and reporting practices for correctly addressing the limitations and improving the comparability of DLS in nanoparticle size measurements.

\section{Materials and methods}

\section{Reference nanoparticles}

Polystyrene latex (PSL), silica, and gold nanoparticles were used in this study. SRMs 1963, 1963a, and 1964, PSL nanoparticles with NIST-certified sizes of 100.7, 101.8 , and $60.39 \mathrm{~nm}$, respectively, were obtained from NIST. Additional PSL nanosphere size standards 3100A, 3070A, 3050A, and 3030A, with reference sizes of $100 \mathrm{~nm}, 70 \mathrm{~nm}, 46 \mathrm{~nm}$, and $31 \mathrm{~nm}$, respectively, were obtained from Thermo Scientific ${ }^{1}$. European Reference Material, ERM®-FD100 and ERM-FD304, silica nanoparticle reference materials with certified sizes of 19.0 and $42.1 \mathrm{~nm}$, respectively, were provided by the Institute for Reference Materials and Measurements (IRMM). Negatively charged, citrate-stabilized gold nanoparticles with nominal sizes of 5, 10, 30, 40, 50, $60,80,100,150,200$, and $250 \mathrm{~nm}$ were obtained from Ted Pella Inc., Redding CA, USA.

\section{Polyplex nanoparticles}

The polyplex nanoparticles were prepared as reported in Ref. (Farkas et al. 2019). Oligo DNA was complexed with synthetic polycationic branched polyethylenimine macromolecules through electrostatic interactions.

Dynamic light scattering

DLS measurements were performed using a ZetaSizer Nano-ZS (Malvern Instruments) (Zetasizer nano user manual 2013). Each sample was loaded into a disposable micro cuvette and measured at $25{ }^{\circ} \mathrm{C}$. The $z$-average size was obtained using the cumulant analysis with a repeatability of $1.6 \%$. Intensity-weighted size distributions were obtained by the regularized non-negatively constrained least-squares (NNLS) method. In the NNLS inversion process, finding the original distribution is mathematically ill-posed since many different distributions produce indistinguishable autocorrelation functions (ACF) given even a small amount of noise. The mathematical problem is addressed by the use of a regularizer parameter, which estimates the level of noise in the ACF and controls the degree of smoothness in the distribution, but this does not solve the distribution indeterminacy. Regularizer values of 0.01 and 0.001 are used for the default "general purpose" and the "multiple narrow modes" algorithms, respectively.

From the NNLS intensity-weighted distribution, the intensity-weighted arithmetic and harmonic diameters may be calculated directly as the arithmetic and harmonic averages of the logarithmically scaled discrete data as long as the varying bin size is taken into consideration.

\footnotetext{
${ }^{1}$ Disclaimer: certain commercial equipment, instruments, or materials are identified in this paper in order to adequately specify the experimental procedure. Such identification does not imply recommendation or endorsement by NIST, nor does it imply that the materials or equipment identified are necessary the best available for the purpose.
} 
Alternatively, the discrete NNLS data may be transformed to a density distribution first and fitted with a lognormal function of $f(x)=\frac{\exp \left(-\frac{1}{2}\left(\frac{\ln x-\mu}{\sigma}\right)^{2}\right)}{x \sigma \sqrt{2 \pi}}$ where $\exp (\mu)$ and $\sigma$ are the scale-parameter and shape-parameter, respectively. The arithmetic and harmonic means are then calculated as $\exp \left(\mu+0.5 \sigma^{2}\right)$ and $\exp (\mu-$ $0.5 \sigma^{2}$ ), respectively (ISO 9276-2:2014). The mathematical convenience of the lognormal distribution function, to which the NNLS size distribution conforms well, is that the transformations between means possess a scalefactor of $\exp \left(0.5 \sigma^{2}\right)$. For example, the intensityweighted harmonic mean is obtained from the intensity-weighted arithmetic mean by dividing the latter by $\exp \left(\sigma^{2}\right)$. Transformation from number- to volume- or volume- to intensity-weighting of respective means requires a multiplication by $\exp \left(3 \sigma^{2}\right)$.

\section{Scanning electron microscopy}

Well-dispersed individual nanoparticles are attached to a substrate by optimizing the surface zeta potential of the substrate and the concentration of the nanoparticle suspensions. In particular, a $20-\mu \mathrm{L}$ droplet of the reference nanoparticle suspension was deposited onto polyL-lysine-coated silicon and incubated for between 1 and $30 \mathrm{~min}$. Samples were immersed in water to remove unattached particles, dried with air, and imaged in a vacuum. Particle size by SEM is reported as the areaequivalent diameter. Imaging was performed on uncoated samples using an FEI Helios Dual-Beam SEM. A working distance of $4 \mathrm{~mm}$, beam current of $0.34 \mathrm{nA}$, and accelerating voltage of 5 or $15 \mathrm{keV}$ were used to obtain all images. The magnification of the instrument was calibrated using a VLSI Standards NanoLattice as a pitch standard, which had been previously calibrated with NIST's Calibrated Atomic Force Microscope. In addition to repeatability of replicate measurements of the area-equivalent diameter values, other uncertainty components arise from the choice of the thresholding level for determining the particle boundary, nonuniformity of the background (which affects the threshold-determination operation), digitization of the particle projection area and signal intensity, and beam alignment and instrument stage instabilities. The combined standard uncertainty is estimated as the square root of the sum of the squares of all the individual uncertainty components (Montoro Bustos et al. 2018).
The expanded uncertainty calculated at the $95 \%$ confidence interval for the SEM size measurements is 3.2\%.

Atomic force microscopy

Samples were prepared on poly-L-lysine-coated mica in the same manner as prepared for SEM. AFM images of the samples were acquired under ambient conditions. Size information of particles is typically obtained from AFM height data. The details of the AFM size measurement of polyplex and polystyrene nanoparticles are described in Ref. (Farkas et al. 2019) and Ref. (Dagata et al. 2016). AFM imaging was performed in TappingMode using Veeco OTESP cantilevers with a Veeco MultiMode AFM and Nanoscope IV controller. Calibration of the height was performed using a set of NANO2 step height reference standards over the range of 7 to $700 \mathrm{~nm}$. Particle size analysis using AFM image data was performed using resources available in Nanoscope Version 5. A first-order image flattening routine is applied to obtain a global background suitable for obtaining particle height measurements. Particles in each image are identified by visual inspection and excluded before the background is calculated. No filtering of the data was performed. In addition to repeatability of replicate measurements of the modal height values, other major uncertainty components arise from particle-substrate and particle-tip deformations, background flatness, and calibration. The combined standard uncertainty is estimated as the square root of the sum of the squares of all the individual uncertainty components. The expanded uncertainty calculated at the $95 \%$ confidence interval for the AFM height measurements is $2.8 \%$.

Acknowledgements The authors wish to thank George W. Mulholland (NIST) for providing the SRM 1963 reference material, and András E. Vladár (NIST) and Prem Kavuri (NIST) for their assistance with SEM imaging. The authors also acknowledge insightful discussions with John A. Dagata (NIST).

Author contribution N.F. conceived the study and performed the measurements. N.F. and J.A.K. analyzed the results and prepared the manuscript.

Funding The research leading to these results received funding from the NIST Measurement Science and Engineering Research Grant Programs under Grant Agreement No. 70NANB17H294. 
Data availability The datasets generated during the current study are available from the corresponding author on reasonable request.

Code availability Not applicable.

\section{Declarations}

Conflict of interest The authors declare no competing interests.

Open Access This article is licensed under a Creative Commons Attribution 4.0 International License, which permits use, sharing, adaptation, distribution and reproduction in any medium or format, as long as you give appropriate credit to the original author(s) and the source, provide a link to the Creative Commons licence, and indicate if changes were made. The images or other third party material in this article are included in the article's Creative Commons licence, unless indicated otherwise in a credit line to the material. If material is not included in the article's Creative Commons licence and your intended use is not permitted by statutory regulation or exceeds the permitted use, you will need to obtain permission directly from the copyright holder. To view a copy of this licence, visit http://creativecommons.org/licenses/by/4.0/.

\section{References}

ASTM E2490-09:2015, Standard guide for measurement of particle size distribution of nanomaterials in suspension by photon correlation spectroscopy (PCS)

ASTM E3247-20:2020, Standard test method for measuring the size of nanoparticles in aqueous media using dynamic light scattering

Barberio AE, Smith SG, Correa S, Nguyen C, Nhan B, Melo M, Tokatlian T, Suh H, Irvine DJ, Hammond PT (2020) Cancer cell coating nanoparticles for optimal tumor-specific cytokine delivery. ACS Nano 14:11238-11253. https://doi. org/10.1021/acsnano.0c03109

Bazylińska U (2017) Rationally designed double emulsion process for co-encapsulation of hybrid cargo in stealth nanocarriers. Colloid Surf A 532:476-482. https://doi.org/10.1016/j. colsurfa.2017.04.027

Bhattacharjee S (2016) DLS and zeta potential — what they are and what they are not? J Control Release 235:337-351. https://doi.org/10.1016/j.jconrel.2016.06.017

Chen G, Abdeen AA, Wang Y, Shahi PK, Robertson S, Xie R, Suzuki M, Pattnaik BR, Saha K, Gong S (2019) A biodegradable nanocapsule delivers a Cas9 ribonucleoprotein complex for in vivo genome editing. Nat Nanotechnol 14: 974-980. https://doi.org/10.1038/s41565-019-0539-2

Chen F, Wang G, Griffin JI, Brenneman B, Banda NK, Holers VM, Backos DS, Wu LP, Moghimi SM, Simberg D (2017) Complement proteins bind to nanoparticle protein corona and undergo dynamic exchange in vivo. Nat Nanotechnol 12: 387-393. https://doi.org/10.1038/nnano.2016.269
Coleman VA, Jämting ÅK, Catchpoole HJ, Roy M, Herrmann J (2011) Nanoparticles and metrology: a comparison of methods for the determination of particle size distributions. Instrumentation, Metrology, and Standards for Nanomanufacturing, Optics, and Semiconductors V. Proc SPIE 8105:810504. https://doi.org/10.1117/12.894297

D'Mello SR et al (2017) The evolving landscape of drug products containing nanomaterials in the United States. Nat Nanotechnol 12:523-529. https://doi.org/10.1038 /nnano.2017.67

Dagata JA et al (2016) Method for measuring the diameter of polystyrene latex reference spheres by atomic force microscopy. Special Publication (NIST SP) 260-185 https://doi. org/10.6028/NIST.SP.260-185

DeLoid GM, Cohen GM, Pyrgiotakis P, Demokritou P (2017) An integrated dispersion preparation, characterization and in vitro dosimetry methodology for engineered nanomaterials. Nat Protoc 12:355-371. https://doi. org/10.1038/nprot.2016.172

Di Francesco T, Borchard G (2018) A robust and easily reproducible protocol for the determination of size and size distribution of iron sucrose using dynamic light scattering. J Pharmaceut Biomed 152:89-93. https://doi.org/10.1016/j. jpba.2018.01.029

Farkas N, Scaria PV, Woodle MC, Dagata JA (2019) Physicalchemical measurement method development for self-assembled, core-shell nanoparticles. Sci Rep 9:1655. https://doi. org/10.1038/s41598-018-38194-y

Hanus LH, Ploehn HJ (1999) Conversion of intensity-averaged photon correlation spectroscopy measurements to numberaveraged particle size distributions. 1. Theoretical development. Langmuir 15:3091-3100. https://doi.org/10.1021 /la980958w

Hess WF (2004) Representation of particle size distributions in practice. Chem Eng Technol 27:624-629. https://doi. org/10.1002/ceat.200403188

Institute for Reference Materials and Measurements of the European Commission's Joint Research Centre, Certificate of analysis ERM-FD304 (2012)

ISO 22412:2017, Particle size analysis - dynamic light scattering (DLS)

ISO 9276-1:1998, Representation of results of particle size analysis - part 1: graphical representation

ISO 9276-2:2014, Representation of results of particle size analysis - part 2: calculation of average particle sizes/ diameters and moments from particle size distributions

Johnston ST, Faria M, Crampin EJ (2018) An analytical approach for quantifying the influence of nanoparticle polydispersity on cellular delivered dose. J R Soc Interface 15:20180364. https://doi.org/10.1098/rsif.2018.0364

Kiss LB, Söderlund J, Niklasson GA, Granqvist CG (1999) New approach to the origin of lognormal size distributions of nanoparticles. Nanotechnology 10:25-28. https://doi. org/10.1088/0957-4484/10/1/006

Koppel DE (1972) Analysis of macromolecular polydispersity in intensity correlation spectroscopy: the method of cumulants. J Chem Phys 57:4814-4820. https://doi.org/10.1063 $/ 1.1678153$

Liu Z, Chen M, Guo Y, Wang X, Zhang L, Zhou J, Li H, Shi Q (2019) Self-assembly of cationic amphiphilic cellulose-g- 
poly (p-dioxanone) copolymers. Carbohydr Polym 204:214 222. https://doi.org/10.1016/j.carbpol.2018.10.020

Maguire CM, Rösslein M, Wick P, Prina-Mello A (2018) Characterisation of particles in solution - a perspective on light scattering and comparative technologies. Sci Technol Adv Mater 19:733-745. https://doi.org/10.1080 /14686996.2018.1517587

Montoro Bustos AR, Purushotham KP, Possolo A, Farkas N, Vladár AE, Murphy KE, Winchester MR (2018) Validation of single particle ICP-MS for routine measurements of nanoparticle size and number size distribution. Anal Chem 90: 14376-14386. https://doi.org/10.1021/acs.analchem.8 b03871

Myerson JW, Braender B, Mcpherson O, Glassman PM, Kiseleva RY, Shuvaev VV, Marcos-Contreras O, Grady ME, Lee HS, Greineder CF, Stan RV, Composto RJ, Eckmann DM, Muzykantov VR (2018) Flexible nanoparticles reach sterically obscured endothelial targets inaccessible to rigid nanoparticles. Adv Mater 30:1802373. https://doi.org/10.1002 /adma.201802373

National Institute of Standards and Technology (2003) Certificate of Analysis Standard Reference Material 1963

Nicolas J, Liu S, Zhao D, Caruso F, Reichmanis E, Buriak JM (2018) Best practices for new polymers and nanoparticulate systems. Chem Mater 30:6587-6588. https://doi.org/10.1021 /acs.chemmater.8b02088

Niebel Y, Buschmann MD, Lavertu M, De Crescenzo G (2014) Combined analysis of polycation/ODN polyplexes by analytical ultracentrifugation and dynamic light scattering reveals their size, refractive index increment, stoichiometry, porosity, and molecular weight. Biomacromolecules 15: 940-947. https://doi.org/10.1021/bm4018148
Nobbmann U, Morfesis A (2009) Light scattering and nanoparticles. Mater Today 12:52-54. https://doi.org/10.1016/S13697021(09)70164-6

Takechi-Haraya Y, Goda Y, Sakai-Kato K (2018) Imaging and size measurement of nanoparticles in aqueous medium by use of atomic force microscopy. Anal Bioanal Chem 410: 1525-1531. https://doi.org/10.1007/s00216-017-0799-3

Thomas JC (1987) The determination of log normal particle size distributions by dynamic light scattering. J Colloid Interface Sci 17:187-192. https://doi.org/10.1016/0021-9797(87 )90182-2

Ulasov AV, Khramtsov YV, Trusov GA, Rosenkranz AA, Sverdlov ED, Sobolev AS (2011) Properties of PEI-based polyplex nanoparticles that correlate with their transfection efficacy. Mol Ther 19:103-112. https://doi.org/10.1038 /mt.2010.233

Xu M, Shen J, Thomas JC, Huang Y, Zhu X, Clementi LA, Vega JR (2018) Information-weighted constrained regularization for particle size distribution recovery in multiangle dynamic light scattering. Opt Express 26:15-31. https://doi. org/10.1364/OE.26.000015

Yeap SP, Lim J, Ngang HP, Ooi BS, Ahmad AL (2018) Role of particle-particle interaction towards effective interpretation of z-average and particle size distributions from dynamic light scattering (DLS) analysis. J Nanosci Nanotechnol 18: 6957-6964. https://doi.org/10.1166/jnn.2018.15458

Zetasizer nano user manual, Malvern Instruments (2013)

Publisher's note Springer Nature remains neutral with regard to jurisdictional claims in published maps and institutional affiliations. 